\title{
Jackstone Calculus: A Rare Subtype of Urinary Stone with a Sea-Urchin Appearance
}

\author{
Barbara Brogna ${ }^{1,2 *}$, Federica Flammia², Francesco Ciriaco Flammia² and Ugo Flammia1,2 \\ ${ }^{1}$ Institution of Radiology at 'University of study of Campania, Luigi Vanvitelli', piazza Miraglia 2, Napoli, Italy \\ ${ }^{2}$ Radiological center CEDIR, via Cristofaro Colombo n 10, Grottaminarda 83035, Avellino, Italy
}

*Corresponding author: Barbara Brogna, Institution of Radiology at 'University of study of Campania, Luigi Vanvitelli', piazza Miraglia 2, Napoli, Italy, E-mail: barbara.brogna@unicampania.it

Received: 13 Nov, 2018 | Accepted: 13 Dec, 2018 | Published: 19 Dec, 2018

Citation: Brogna B, Flammia F, Flammia FC, Flammia U (2018) Jackstone Calculus: A Rare Subtype of Urinary Stone with a Sea-Urchin Appearance. Int J Nephrol Kidney Fail 4(5): dx.doi.org/10.16966/2380-5498.166

Copyright: (C) 2018 Brogna B, et al. This is an open-access article distributed under the terms of the Creative Commons Attribution License, which permits unrestricted use, distribution, and reproduction in any medium, provided the original author and source are credited.

\section{To the Editor,}

We read with interest the article of Wang et al. [1] on Hypocitraturic Nephrolitiasis and stone recurrence. It's known that there are different subtypes of stones. Renal calculi have been extensively described in the literature search, though bladder calculi have been not studied as that of renal calculi. Jackstone calculi are urinary stones with a specific appearance. They are typically found in the bladder and commonly reported in veterinary literature [2,3]. A Jackstone calculus can be found in the upper urinary tract, but it is less common [4]. Few cases of Jackstone calculi were reported in literature. These stone subtypes usually have sharp edges like a sea-urchin or star. This shape also resembles toy jacks [2-8]. They are made up of calcium oxalate dehydrate with a crystalline structure, which makes them easy to fragment during lithotripsy. Therefore, recognizing the characteristic shape of a Jackstone calculus can have clinical and therapeutic implications.

Large bladder calculi also represent a rare condition and are found in 5\% of cases and the first description was found in Egyptian archives $[9,10]$. In our case, a large Jackstone calculus in an 84-yearold-man, who suffered from benign prostatic hypertrophy and nonspecific abdominal pain, was incidentally detected on a Computed Tomography (CT) scan. A stone of about $3 \mathrm{~cm}$ with a speculated shape characterized by a central nucleus with an irregular shape was found in the bladder (Figure 1). The appearance of the stone was compatible with a Jackstone calculus (Figure 2).

Bladder stones rarely formed spontaneously. Male are more affected than female. Bladder obstruction remains the most common risk factor for bladder calculi [2-6].

Other causes are prostatic pathology, some medications, surgery to the urinary tract, metabolic abnormalities and urinary infections $[2,3,6,9]$. Complications include bladder adherence with chronic inflammation of the wall.

In women bladder calculi are secondary to female pelvic surgery, genital prolapse and foreign bodies, in children bladder calculi have high occurrence in male in development countries and are associate to Nutritional deficiencies [9].

Most of bladder calculi are mobile, though stones formed on a suture or retained stent or on the intravesical portion of a papillary tumor are fixed. Generally, most of urinary calculi are calcium oxalate dehydrate monohydrate stones with a regular, homogeneous appearance and smooth margin. During endoscopic examinations they appear black, whereas Jackstone calculi are irregular and yellow [2]. Calcium oxalate dehydrate monohydrate stones are less susceptible to fragmentation through lithotripsy.

Stones composed by acid uric are also common in the bladder. Bladder stones made of struvite or calcium phosphate are typically found in patients with spinal cord injuries.

The typical Jackstone calculus grows at the peripheries, which touch the wall causing the precipitation of mucoproteins from the bladder wall that allows the deposition of more calcium oxalate in the central core. This explains its shape [2-8]. Jackstone calculi on imaging modality have been extensively described in animals as cattle, cats and dogs. They were typically found in dogs with silica compositions. Silica Jackstone calculi are rare in humans and occur in only $0.02 \%$ of cases [2]. These calculi have low radio density on plain $\mathrm{x}$-ray. Only one case of Jackstone calculi with silica compositions were previously described in a human patient [11]. We are able to find 5 recent reports of Jackstone calculi described on imaging in patients who suffered of prostatic hypertrophy $[2,3,6-8]$. The diagnosis was usually made incidentally. In adults, the classical treatment for bladder calculi is endoscopic transurethral disintegration with mechanical cystolithotripsy, ultrasound and electro hydraulic lithotripsy. Stones too large (greater than $4 \mathrm{~cm}$ ) or with hard structure are treated with open cystolithotomy or pneumatic lithotripsy. Open surgery is the main treatment for bladder calculi in children $[9,10]$.

Imaging studies include abdominal plain x-ray, ultrasound, transrectal ultrasound and abdominal CT. Stone shape can have effects on the interaction of shock way lithotripsy with a stone. Dretler and 


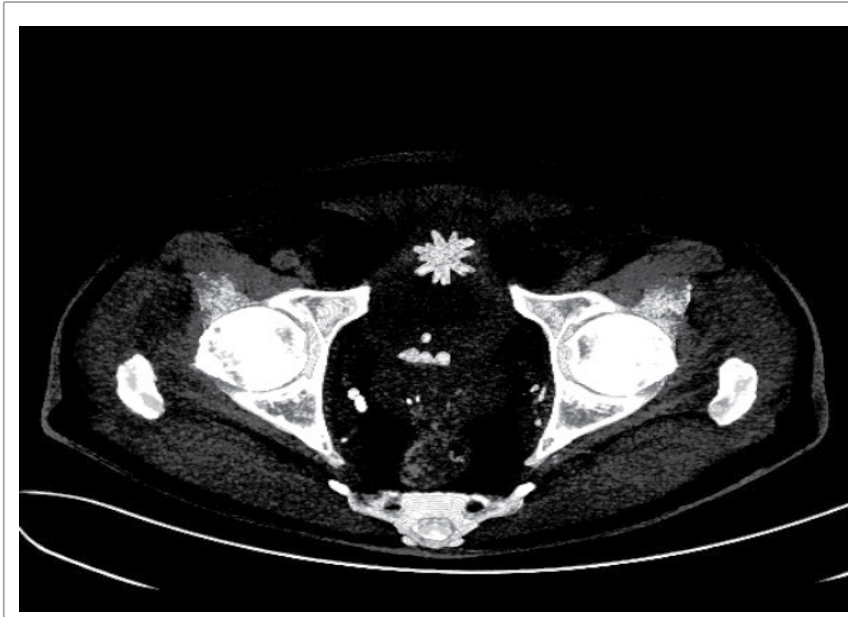

Figure 1: Maximum intensity projection (MIP) reconstruction of the Jackstone calculus in the bladder.

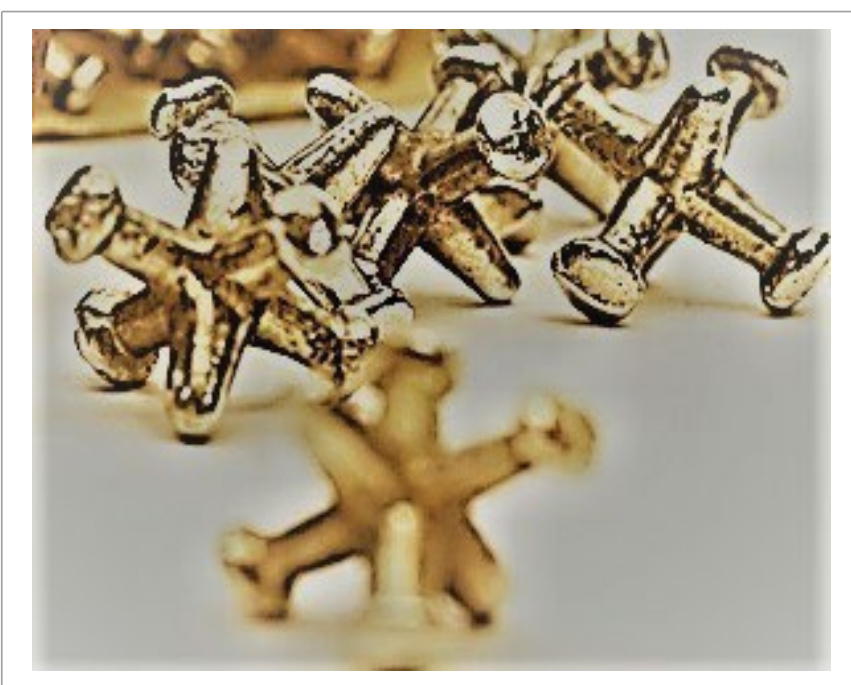

Figure 2: Picture of a Jack's toy.

Polykoff [12] studied the fragility of different calcium oxalate stones by their shape on plain $\mathrm{x}$-ray.

Helical computed tomography (CT) has been proposed the most accurate diagnostic tool to predict the success or failure of lithotripsy and plays a pivotal rolein the diagnosis. Numerous studies showed that it can predict stones chemical content through the Hounsfield unit (HU) analysis. Nevertheless the study of Zarse et al. [13] assessed that stone fragility correlated more with their appearance than with Hounsfield analysis. However the dual energy CT has improved stone characterization and can accurately assess stone composition with stone morphology [14]. It is important to recognize stone appearance such as some stones could be resistant to lithotripsy and CT diagnosis could be used to avoid the patient to an ineffective procedure. Léger et al. [15] described that stones organized in crystalline structure broke more easily than those that were less organized.

Furthermore, it's important to recognize bladder calculi through clinical and radiological evaluations because they can detect other pathologies and metabolic anomalies and for the clinical management.
Funding: No funding was received for the publication

\section{Conflicts of Interest}

The authors do not have any disclosures to report.

\section{Ethical Approval}

For this type of the study formal consent is not required.

\section{References}

1. Wang CJ, Hsu CS, Chen HW, Tsai PC, Chang CH (2016) Long-Term Effects of Lemonade Therapy on Hypocitraturic Nephrolithiasis and Stone Recurrence: A Mini Review. Int J Nephrol Kidney Failure 2.

2. Perlmutter S, Hsu CT, Villa PA, Katz DS (2002) Sonography of a human jackstone calculus. J Ultrasound Med 21: 1047-1051.

3. Singh KJ, Tiwari A, Goyal A (2011) Jackstone: A rare entity of vesical calculus. Indian J Urol 27: 543-544.

4. Grases F, Costa-Bauza A, Prieto RM, Saus C, Servera A, et al. (2011) Rare calcium oxalate monohydrate calculus attached to the wall of the renal pelvis. Int J Urol 18: 323-325.

5. Sweeney AP, Dyer RB (2015) The" jackstone" appearance. Abdom Imaging 40: 2906-2907.

6. Roose R, Feyaerts F (2018) Jackstone Calculus: A Spiky Cause of Haematuria. J Belg Soc Radiol 102: 37.

7. Rivell C, Coren J (2012) Onesies, Twosies, not a game of Jackstones. Osteopathic Family Physician 4: 88-90.

8. Subasinghe D, Goonewardena S, Kathiragamathamby V (2017) Jack stone in the bladder: case report of a rare entity. BMC Urol 17: 40.

9. Schwartz BF, Stoller ML (2000) The vesical calculus. Urol Clin North Am 27: 333-346.

10. Papatsoris AG, Varkarakis I, Dellis A, Deliveliotis C (2006) Bladder lithiasis: from open surgery to lithotripsy. Urol Res 34: 163-167.

11. Levison DA, Crocker PR, Banim S, Wallace DM (1982) Silica stones in the urinary bladder. Lancet 1: 704-705.

12. Dretler SP, Polykoff G (1996) Calcium oxalate stone morphology: fine tuning our therapeutic distinctions. J Urol 155: 828-833.

13. Zarse $C A$, Hameed $T A$, Jackson ME, Pishchalnikov $Y A$, Lingeman JE, et al. (2007) CT visible internal stone structure, but not Hounsfield unit value, of calcium oxalate monohydrate (COM) calculi predicts lithotripsy fragility in vitro. Urol Res 35: 201-206.

14. Primak AN, Fletcher JG, Vrtiska TJ, Dzyubak OP, Lieske JC, et al. (2007) Noninvasive differentiation of uric acid versus non-uric acid kidney stones using dual-energy CT. Acad Radiol 14: 1441-1447.

15. Léger $P$, Daudon $M$, Magnier M. (1990) Expérience in vitro de lithotripsiepyézo-électrique à repérageultra-sonique sur le lithotripteur EDAP LT 01. Journal d'Urologie 96: 353. 